\title{
Effect of Different Varieties, Media and Storage Temperature on in vitro Pollen Germination in Mango (Mangifera indica L.)
}

\author{
D. N. Dhamsaniya*, D. K. Varu and D. R. Mehta \\ College of Horticulture, Junagadh Agricultural University, Junagadh, Gujarat, India \\ *Corresponding author
}

\author{
A B S T R A C T
}

\begin{tabular}{l} 
Ke y w or d s \\
Mango; Pollen \\
storage, In vitro \\
pollen germination, \\
$\begin{array}{l}\text { Storage media, } \\
\text { Storage temperature }\end{array}$ \\
\hline Article Info \\
$\begin{array}{l}\text { Accepted: } \\
\text { 07 September } 2020 \\
\text { Available Online: } \\
\text { 10 October } 2020\end{array}$ \\
\hline
\end{tabular}

A study was conducted to investigate in vitro pollen germination of four mango varieties, viz. Kesar, Alphonso, Mallika and Dudhpendo. Pollen grains were stored in three different media (n-hexane, paraffin oil and without media) under four different storage temperature $\left(-20^{\circ} \mathrm{C},-4^{0} \mathrm{C}, 4^{0} \mathrm{C}\right.$ and room temperature). The experiment was laid out in Completely Randomized Design (CRD) with factorial concept. Germination media having $10 \%$ sucrose +100 ppm $\mathrm{H}_{3} \mathrm{BO}_{3}+300 \mathrm{ppm} \mathrm{Ca}\left(\mathrm{NO}_{3}\right)_{2}+200$ ppm $\mathrm{MgSO}_{4}+100$ ppm $\mathrm{KNO}_{3}$ used for in vitro pollen germination for all the varieties. Variation due to different varieties, media and temperature was found significant. Among four varieties, maximum germination was recorded in Kesar during 7, 14, 21, 28, 35, 42 and 56 days of storage, whereas, in Mallika at 49 days of storage. In storage media, n-hexane found best for retaining more germination ability during all storage days. Room temperature storage of pollen showed no germination from 21 days of storage. Maximum pollen germination was recorded at $-20^{\circ} \mathrm{C}$ followed by $-4^{0} \mathrm{C}$. On the basis of interaction effect of results obtained, it could be concluded that mango pollen grains of Kesar and Mallika could be stored successfully up to 56 days in n-hexane at $-20^{\circ} \mathrm{C}$.

\section{Introduction}

Mango (Mangifera indica $\mathrm{L}$.) belongs to the family Anacardiaceae. It is the most important crop among the tropical and subtropical fruit crops grown in more than 110 countries across the world. Mango $(2 n=40)$, is an allopolyploid, most probably amphidiploid and cross pollinated species.

Individual mango flower is small, ranging in size from five to ten $\mathrm{mm}$ in diameter. The three to nine (usually five) cream-coloured petals typically change their colour before falling (Naik and Rao, 1943). Anthers are around $1.2 \mathrm{~mm}$ long, usually four-lobed, and dehiscence occurs longitudinally to reveal pollen grains (Scholefield, 1982). The ovary has one chamber that contains only one ovule (Free, 1993) and superior, sessile, free, yellow- coloured (Kosterman and Bompard, 1993; Galan-Sauco, 1999). Morphologically, pollen grains are $20-45 \mu \mathrm{m}$ long, tricolpate and generally have three apertures along the longitudinal sides when they dry (Singh, 1961; Davenport, 2009).

Mango pollination is a complex physiological 
phenomenon. It initiates with the contact of pollen with the stigmatic surface of the style. Pollen adhesion to stigmatic surface differs from cultivar to cultivar (Dutta et al., 2013). Stigma receptivity commenced about $18 \mathrm{hrs}$ prior to anthesis and continued for $72 \mathrm{~h}$ after anthesis and is most receptive during the first 6 hrs in mango (Pimentel et al., 1984; Iyler and Scnell, 2009). After pollen deposition on the stigma, pollen tube germination and growth is the next step in the process of fruit setting. The minimum time required for pollen germination is $1.5 \mathrm{hrs}$ in mango (Sen et al., 1946; Singh, 1954; Spencer and Kennard, 1955). Depending upon ambient temperature, the rate of pollen germination differs among cultivars.

Pollen viability and its germination are cultivar dependent characters in mango (Singh, 1954; Abourayya et al., 2011). Mango pollen requires some specific conditions of temperature and humidity as well as an artificial growth medium to achieve in vitro germination equaling that of pollen on stigmas in vivo (De Wet and Robbertse, 1986). To fulfil the above objective, an attempt was made to determine the in vitro pollen germination of different mango cultivars under different storage media and storage temperature for mango pollen storage.

\section{Materials and Methods}

\section{Pollen collection and drying}

Four mango varieties viz., Kesar, Alphanso, Dudhpendo and Mallika were chosen for present study available at Fruit Research Station, Sakkarbaug, Junagadh Agricultural University, Junagadh. The pollen used in this experiment was collected from freshly opened flowers of all four mango cultivars between 8:00 and 10:00 am $\left(\leq 23^{\circ} \mathrm{C}\right)$.

The harvested flowers were placed in the sun to prompt anther dehiscence. Pollen grains visible on the anthers as a greyish powder, were collected in a petri dish using forceps and dried in a desiccator for two hours.

\section{Pollen storage}

Dried pollen grains were suspended in the different three storage media, viz. n-hexane, paraffin oil and without media and stored at room temperature in a controlled growth room $\left(27^{\circ} \mathrm{C}\right)$ and at low temperatures in a cold-storage facility $\left(4^{\circ} \mathrm{C},-4^{\circ} \mathrm{C},-20^{\circ} \mathrm{C}\right)$ at the Post Graduate Lab, College of Horticulture, Junagadh.

\section{Sample preparation}

The samples of pollen grains were taken from storage each week for observations. Pollen grains were separated from oil/organic solvent via a filtration process. Paraffin oil along with pollen grains was poured onto the filter paper directly from the petri dishes and allowed to filter through, leaving pollen grains on the paper. The pollen grains on the filter paper were then rinsed 4-5 times with n-hexane to remove residual oil, before they were dried for 10-15 minutes.

\section{In vitro pollen germination}

Germination media with different concentrations of sucrose, $\mathrm{H}_{3} \mathrm{BO}_{3}, \mathrm{Ca}\left(\mathrm{NO}_{3}\right)_{2}$, $\mathrm{MgSO}_{4}$ and $\mathrm{KNO}_{3}$ was analyzed for in vitro pollen germination for all the varieties. Germination tests were incubated at $27 \pm 2^{\circ} \mathrm{C}$ for 6 hours. Pollen grains were considered as germinated when pollen tube length surpassed their diameter. Germination media having $10 \%$ sucrose $+100 \mathrm{ppm}_{3} \mathrm{BO}_{3}+300 \mathrm{ppm}$ $\mathrm{Ca}\left(\mathrm{NO}_{3}\right)_{2}+200 \mathrm{ppm} \mathrm{MgSO}_{4}+100 \mathrm{ppm}$ $\mathrm{KNO}_{3}$ was found best and was further used for in vitro pollen germination for all the varieties during the research program. 


\section{Statistical analysis}

Statistical analysis of data of various characters was carried out as per Completely Randomized Design (Factorial). Analysis of variance was worked out using standard statistical procedures as described by Panse and Sukhatme (1985).

\section{Results and Discussion}

\section{Effect of variety on in vitro pollen germination}

Pollen germination is the most important parameter for long term pollen storage and its use for pollination. The variation due to varieties in pollen germination was found significant for all storage days. Significantly maximum pollen germination $(53.95,43.64$, $31.62,23.06,16.04,11.34 \& 0.95 \%$ ) was recorded in Kesar $\left(\mathrm{V}_{1}\right)$ followed by Mallika $\left(\mathrm{V}_{3}\right)$ during 7, 14, 21, 28, 35, 42 and 56 days. Whereas at 49 days of storage, maximum pollen germination $(4.13 \%)$ was noted in Mallika $\left(\mathrm{V}_{3}\right)$ followed with $\operatorname{Kesar}\left(\mathrm{V}_{1}\right)$. During all storage days, lowest pollen germination was observed in variety Alphonso $\left(\mathrm{V}_{2}\right)$ (Table 1).

\section{Effect of media on in vitro pollen germination}

The differences observed in pollen germination due to storage media was also found significant and maximum pollen germination $(63.71,52.63,41.76,32.64$, $24.21,15.99,6.65$ and $1.95 \%)$ was registered in pollen grains stored with media n-hexane $\left(\mathrm{M}_{1}\right)$ followed by paraffin oil $\left(\mathrm{M}_{2}\right)$ during all storage days. Similarly, lowest pollen germination $(31.75,21.11,7.26$ and $1.37 \%)$ were found in pollen grains stored without any media $\left(\mathrm{M}_{3}\right)$ at $7,14,21$ and 28 days, respectively. From 35 days of storage, it was recorded to zero (Table 1).

\section{Effect of storage temperature on in vitro} pollen germination

In the present study, wide variation in germination of pollen grains was observed significant with different storage temperatures and durations among different mango cultivars. Maximum pollen germination (70.94, 60.30, 45.64, 33.24, 23.32, 16.99, 9.40 and $2.60 \%$ ) was noted in pollen grains stored at temperature $-20^{\circ} \mathrm{C}\left(\mathrm{S}_{1}\right)$ followed by $-4^{\circ} \mathrm{C}$ $\left(\mathrm{S}_{2}\right)$ during all storage days, respectively. However, minimum pollen germination was found in pollen grains stored at room temperature $\left(S_{4}\right)$ for all storage days.

Interaction effect of variety and media on in vitro pollen germination

Variation due to interaction effect of variety and media on pollen germination was found significant at all days of storage. Maximum pollen germination $(68.49,59.98,45.55$, $37.22,27.34,20.47,8.87 \& 2.86 \%$ ) was observed in treatment combination $\mathrm{V}_{1} \mathrm{M}_{1}$ at all days of storage followed by $\mathrm{V}_{3} \mathrm{M}_{1}$ at 7, 14, $21,35,42$ and 49 as well as by $\mathrm{V}_{4} \mathrm{M}_{1}$ at 28 and 56 days. Lowest pollen germination was noted in $\mathrm{V}_{2} \mathrm{M}_{3}$ at 7 and 21 days of storage. At 14 and 28 days of storage, it was observed in $\mathrm{V}_{3} \mathrm{M}_{3}$. Pollen germination exerted zero in no media (control) with all varieties i.e. treatment combination $\mathrm{V}_{1} \mathrm{M}_{3}, \mathrm{~V}_{2} \mathrm{M}_{3}, \mathrm{~V}_{3} \mathrm{M}_{3}$ and $\mathrm{V}_{4} \mathrm{M}_{3}$ after 35 days of storage (Table 2).

Interaction effect of media and storage temperature on in vitro pollen germination

Variation due to interaction effect of different media and storage temperatures on pollen germination was also found significant at all days of storage. Maximum pollen germination $(85.48,76.42,63.43,51.94,42.05,31.88$, $21.43 \& 7.81 \%$ ) was noted in pollen grains stored with n-hexane at $-20^{\circ} \mathrm{C}\left(\mathrm{M}_{1} \mathrm{~S}_{1}\right)$ at all days of storage, respectively (Table 3 ). 
Table.1 Effect of different mango variety, media and temperature on in vitro pollen germination

\begin{tabular}{|c|c|c|c|c|c|c|c|c|}
\hline \multirow[t]{2}{*}{ Treatments } & \multicolumn{8}{|c|}{ In vitro pollen germination $(\%)$} \\
\hline & 7 & 14 & 21 & 28 & 35 & 42 & 49 & 56 \\
\hline \multicolumn{9}{|l|}{ Factor A (Variety - 4) } \\
\hline $\mathrm{V}_{1}:$ Kesar & 53.95 & 43.64 & 31.62 & 23.06 & 16.04 & 11.34 & 3.97 & 0.95 \\
\hline $\mathrm{V}_{2}:$ Alphonso & 46.66 & 36.79 & 25.15 & 16.41 & 10.38 & 5.88 & 0.89 & 0.00 \\
\hline $\mathrm{V}_{3}:$ Mallika & 52.84 & 39.74 & 29.55 & 21.11 & 15.02 & 8.70 & 4.13 & 0.78 \\
\hline $\mathrm{V}_{4}$ : Dudhpendo & 47.58 & 36.65 & 26.52 & 18.69 & 12.66 & 5.91 & 2.12 & 0.86 \\
\hline S.Em. \pm & 0.27 & 0.24 & 0.25 & 0.21 & 0.20 & 0.12 & 0.05 & 0.01 \\
\hline C.D. at $5 \%$ & 0.75 & 0.67 & 0.71 & 0.60 & 0.56 & 0.34 & 0.15 & 0.03 \\
\hline \multicolumn{9}{|l|}{ Factor B (Media - 3) } \\
\hline $\mathrm{M}_{1}$ : n-hexane & 63.71 & 52.63 & 41.76 & 32.64 & 24.21 & 15.99 & 6.65 & 1.95 \\
\hline $\mathrm{M}_{2}$ : Paraffin oil & 55.32 & 43.87 & 35.61 & 25.44 & 16.37 & 7.88 & 1.69 & 0.00 \\
\hline $\mathrm{M}_{3}:$ No media (Control) & 31.75 & 21.11 & 7.26 & 1.37 & 0.00 & 0.00 & 0.00 & 0.00 \\
\hline S.Em. \pm & 0.23 & 0.21 & 0.22 & 0.19 & 0.17 & 0.11 & 0.05 & 0.01 \\
\hline C.D. at $5 \%$ & 0.65 & 0.58 & 0.61 & 0.52 & 0.49 & 0.30 & 0.13 & 0.03 \\
\hline \multicolumn{9}{|l|}{$\begin{array}{l}\text { Factor C (Storage } \\
\text { Temperature - 4) }\end{array}$} \\
\hline $\mathrm{S}_{1}:-20^{\circ} \mathrm{C}$ & 70.94 & 60.30 & 45.64 & 33.24 & 23.32 & 16.99 & 9.40 & 2.60 \\
\hline $\mathrm{S}_{2}:-4^{\circ} \mathrm{C}$ & 64.12 & 52.15 & 37.88 & 24.81 & 16.26 & 9.43 & 1.72 & 0.00 \\
\hline $\mathrm{S}_{3}:+4^{\circ} \mathrm{C}$ & 54.33 & 42.35 & 29.32 & 21.23 & 14.53 & 5.40 & 0.00 & 0.00 \\
\hline $\mathrm{S}_{4}$ : Room temperature & 11.64 & 2.01 & 0.00 & 0.00 & 0.00 & 0.00 & 0.00 & 0.00 \\
\hline S.Em. \pm & 0.27 & 0.24 & 0.25 & 0.21 & 0.20 & 0.12 & 0.05 & 0.01 \\
\hline C.D. at $5 \%$ & 0.75 & 0.67 & 0.71 & 0.60 & 0.56 & 0.34 & 0.15 & 0.03 \\
\hline \multicolumn{9}{|l|}{ Interactions } \\
\hline \multicolumn{9}{|l|}{$\mathbf{V} \times \mathbf{M}$} \\
\hline S.Em. \pm & 0.46 & 0.41 & 0.44 & 0.37 & 0.35 & 0.21 & 0.09 & 0.02 \\
\hline C.D. at $5 \%$ & 1.30 & 1.15 & 1.22 & 1.04 & 0.97 & 0.59 & 0.26 & 0.05 \\
\hline \multicolumn{9}{|l|}{$\mathbf{V} \times \mathbf{S}$} \\
\hline S.Em. \pm & 0.54 & 0.48 & 0.50 & 0.43 & 0.40 & 0.24 & 0.11 & 0.02 \\
\hline C.D. at $5 \%$ & 1.50 & 1.33 & 1.41 & 1.20 & 1.12 & 0.69 & 0.30 & 0.06 \\
\hline \multicolumn{9}{|l|}{$\mathbf{M} \times \mathbf{S}$} \\
\hline S.Em. \pm & 0.46 & 0.41 & 0.44 & 0.37 & 0.35 & 0.21 & 0.09 & 0.02 \\
\hline C.D. at $5 \%$ & 1.30 & 1.15 & 1.22 & 1.04 & 0.97 & 0.59 & 0.26 & 0.05 \\
\hline \multicolumn{9}{|l|}{$\mathbf{V} \times \mathbf{M} \times \mathbf{S}$} \\
\hline S.Em. \pm & 0.93 & 0.82 & 0.87 & 0.74 & 0.69 & 0.42 & 0.19 & 0.04 \\
\hline C.D. at $5 \%$ & 2.61 & 2.31 & 2.44 & 2.08 & 1.95 & 1.19 & 0.52 & 0.11 \\
\hline C.V. \% & 3.20 & 3.64 & 5.34 & 6.49 & 8.88 & 9.20 & 11.59 & 10.10 \\
\hline
\end{tabular}


Table.2 Interaction effect of variety and media on in vitro pollen germination

\begin{tabular}{|l|c|c|c|c|c|c|c|c|}
\hline \multirow{2}{*}{$\begin{array}{l}\text { Treatments } \\
\mathbf{V} \times \mathbf{M}\end{array}$} & $\mathbf{7}$ & $\mathbf{1 4}$ & $\mathbf{2 1}$ & $\mathbf{2 8}$ & $\mathbf{3 5}$ & $\mathbf{4 2}$ & $\mathbf{4 9}$ & $\mathbf{5 6}$ \\
\hline $\mathrm{V}_{\mathbf{1}} \mathrm{M}_{1}$ & $\mathbf{6 8 . 4 9}$ & $\mathbf{5 9 . 9 8}$ & $\mathbf{4 5 . 5 5}$ & $\mathbf{3 7 . 2 2}$ & $\mathbf{2 7 . 3 4}$ & $\mathbf{2 0 . 4 7}$ & $\mathbf{8 . 8 7}$ & $\mathbf{2 . 8 6}$ \\
\hline $\mathrm{V}_{\mathbf{1}} \mathrm{M}_{2}$ & 60.14 & 46.59 & 38.60 & 28.90 & 20.78 & 13.55 & 3.05 & 0.00 \\
\hline $\mathrm{V}_{\mathbf{1}} \mathrm{M}_{3}$ & 33.23 & 24.34 & 10.72 & 3.08 & 0.00 & 0.00 & 0.00 & 0.00 \\
\hline $\mathrm{V}_{2} \mathrm{M}_{1}$ & 60.32 & 49.31 & 39.68 & 28.24 & 19.35 & 11.95 & 2.67 & 0.00 \\
\hline $\mathrm{V}_{2} \mathrm{M}_{2}$ & 51.42 & 41.28 & 31.61 & 21.00 & 11.79 & 5.68 & 0.00 & 0.00 \\
\hline $\mathrm{V}_{2} \mathrm{M}_{3}$ & 28.23 & 19.77 & 4.16 & 0.00 & 0.00 & 0.00 & 0.00 & 0.00 \\
\hline $\mathrm{V}_{3} \mathrm{M}_{1}$ & 66.01 & 52.68 & 41.99 & 32.52 & 26.38 & 16.70 & $\underline{8.69}$ & 2.35 \\
\hline $\mathrm{V}_{3} \mathrm{M}_{2}$ & 57.37 & 47.06 & 38.39 & 28.38 & 18.69 & 9.40 & 3.71 & 0.00 \\
\hline $\mathrm{V}_{3} \mathrm{M}_{3}$ & 35.15 & 19.47 & 8.26 & 2.41 & 0.00 & 0.00 & 0.00 & 0.00 \\
\hline $\mathrm{V}_{4} \mathrm{M}_{1}$ & 60.02 & 48.54 & 39.82 & 32.59 & 23.75 & 14.82 & 6.37 & 2.59 \\
\hline $\mathrm{V}_{4} \mathrm{M}_{2}$ & 52.34 & 40.54 & 33.85 & 23.49 & 14.22 & 2.90 & 0.00 & 0.00 \\
\hline $\mathrm{V}_{4} \mathrm{M}_{3}$ & 30.39 & 20.86 & 5.88 & 0.00 & 0.00 & 0.00 & 0.00 & 0.00 \\
\hline $\mathrm{S} . \mathrm{Em} . \pm$ & 0.46 & 0.41 & 0.44 & 0.37 & 0.35 & 0.21 & 0.09 & 0.02 \\
\hline C.D. at 5\% & 1.30 & 1.15 & 1.22 & 1.04 & 0.97 & 0.59 & 0.26 & 0.05 \\
\hline C.V. \% & 3.20 & 3.64 & 5.34 & 6.49 & 8.88 & 9.20 & 11.59 & 10.10 \\
\hline
\end{tabular}

Table.3 Interaction effect of media and storage temperature on in vitro pollen germination

\begin{tabular}{|l|c|c|c|c|c|c|c|c|}
\hline \multirow{2}{*}{\begin{tabular}{|} 
Treatments \\
$\mathbf{M} \times \mathbf{S}$
\end{tabular}} & $\mathbf{7}$ & $\mathbf{1 4}$ & $\mathbf{2 1}$ & $\mathbf{2 8}$ & $\mathbf{3 5}$ & $\mathbf{4 2}$ & $\mathbf{4 9}$ & $\mathbf{5 6}$ \\
\hline $\mathrm{M}_{1} \mathrm{~S}_{1}$ & $\mathbf{8 5 . 4 8}$ & $\mathbf{7 6 . 4 2}$ & $\mathbf{6 3 . 4 3}$ & $\mathbf{5 1 . 9 4}$ & $\mathbf{4 2 . 0 5}$ & $\mathbf{3 1 . 8 8}$ & $\mathbf{2 1 . 4 3}$ & $\mathbf{7 . 8 1}$ \\
\hline $\mathrm{M}_{1} \mathrm{~S}_{2}$ & 77.99 & 66.91 & 55.00 & 42.46 & 28.65 & 18.53 & 5.17 & 0.00 \\
\hline $\mathrm{M}_{1} \mathrm{~S}_{3}$ & 69.88 & 61.17 & 48.62 & 36.16 & 26.13 & 13.54 & 0.00 & 0.00 \\
\hline $\mathrm{M}_{1} \mathrm{~S}_{4}$ & 21.49 & 6.02 & 0.00 & 0.00 & 0.00 & 0.00 & 0.00 & 0.00 \\
\hline $\mathrm{M}_{2} \mathrm{~S}_{1}$ & 74.49 & 65.04 & 54.86 & 42.29 & 27.90 & 19.10 & 6.76 & 0.00 \\
\hline $\mathrm{M}_{2} \mathrm{~S}_{2}$ & 69.03 & 59.03 & 48.25 & 31.96 & 20.13 & 9.75 & 0.00 & 0.00 \\
\hline $\mathrm{M}_{2} \mathrm{~S}_{3}$ & 64.32 & 51.40 & 39.34 & 27.52 & 17.46 & 2.66 & 0.00 & 0.00 \\
\hline $\mathrm{M}_{2} \mathrm{~S}_{4}$ & 13.43 & 0.00 & 0.00 & 0.00 & 0.00 & 0.00 & 0.00 & 0.00 \\
\hline $\mathrm{M}_{3} \mathrm{~S}_{1}$ & 52.87 & 39.45 & 18.64 & 5.49 & 0.00 & 0.00 & 0.00 & 0.00 \\
\hline $\mathrm{M}_{3} \mathrm{~S}_{2}$ & 45.33 & 30.49 & 10.38 & 0.00 & 0.00 & 0.00 & 0.00 & 0.00 \\
\hline $\mathrm{M}_{3} \mathrm{~S}_{3}$ & 28.80 & 14.50 & 0.00 & 0.00 & 0.00 & 0.00 & 0.00 & 0.00 \\
\hline $\mathrm{M}_{3} \mathrm{~S}_{4}$ & 0.00 & 0.00 & 0.00 & 0.00 & 0.00 & 0.00 & 0.00 & 0.00 \\
\hline $\mathrm{S} . \mathrm{Em} . \pm$ & 0.46 & 0.41 & 0.44 & 0.37 & 0.35 & 0.21 & 0.09 & 0.02 \\
\hline C.D. at 5\% & 1.30 & 1.15 & 1.22 & 1.04 & 0.97 & 0.59 & 0.26 & 0.05 \\
\hline C.V. \% & 3.20 & 3.64 & 5.34 & 6.49 & 8.88 & 9.20 & 11.59 & 10.10 \\
\hline & & & & & & & & \\
\hline
\end{tabular}


Table.4 Interaction effect of variety and storage temperature on in vitro pollen germination

\begin{tabular}{|c|c|c|c|c|c|c|c|c|}
\hline \multirow{2}{*}{$\begin{array}{l}\text { Treatments } \\
\mathbf{V} \times \mathbf{S}\end{array}$} & \multicolumn{8}{|c|}{ In vitro pollen germination (\%) } \\
\hline & 7 & 14 & 21 & 28 & 35 & 42 & 49 & 56 \\
\hline $\mathrm{V}_{1} \mathrm{~S}_{1}$ & 71.31 & 61.52 & 48.72 & 35.59 & $\underline{25.97}$ & 21.42 & 12.30 & 3.82 \\
\hline $\mathrm{V}_{1} \mathrm{~S}_{2}$ & 65.94 & 57.42 & 42.18 & 30.47 & 17.87 & 14.01 & 3.60 & 0.00 \\
\hline $\mathrm{V}_{1} \mathrm{~S}_{3}$ & 63.50 & 51.27 & 35.60 & 26.20 & 20.32 & 9.93 & 0.00 & 0.00 \\
\hline $\mathrm{V}_{1} \mathrm{~S}_{4}$ & 15.06 & 4.34 & 0.00 & 0.00 & 0.00 & 0.00 & 0.00 & 0.00 \\
\hline $\mathrm{V}_{2} \mathrm{~S}_{1}$ & 69.44 & 59.16 & 43.45 & 29.57 & 18.74 & 12.06 & 3.56 & 0.00 \\
\hline $\mathrm{V}_{2} \mathrm{~S}_{2}$ & 60.03 & 48.90 & 31.92 & 19.08 & 12.02 & 7.63 & 0.00 & 0.00 \\
\hline $\mathrm{V}_{2} \mathrm{~S}_{3}$ & 50.71 & 39.10 & 25.23 & 16.99 & 10.77 & 3.82 & 0.00 & 0.00 \\
\hline $\mathrm{V}_{2} \mathrm{~S}_{4}$ & 6.45 & 0.00 & 0.00 & 0.00 & 0.00 & 0.00 & 0.00 & 0.00 \\
\hline $\mathrm{V}_{3} \mathrm{~S}_{1}$ & 73.49 & $\underline{61.31}$ & 46.54 & 36.97 & 26.49 & 19.62 & 13.23 & 3.14 \\
\hline $\mathrm{V}_{3} \mathrm{~S}_{2}$ & 67.02 & 50.63 & 41.00 & 25.14 & 18.76 & 11.27 & 3.29 & 0.00 \\
\hline$V_{3} S_{3}$ & 55.18 & 43.32 & 30.65 & 22.32 & 14.84 & 3.92 & 0.00 & 0.00 \\
\hline $\mathrm{V}_{3} \mathrm{~S}_{4}$ & 15.69 & 3.68 & 0.00 & 0.00 & 0.00 & 0.00 & 0.00 & 0.00 \\
\hline $\mathrm{V}_{4} \mathrm{~S}_{1}$ & 69.54 & 59.22 & 43.86 & 30.83 & 22.05 & 14.89 & 8.50 & 3.45 \\
\hline $\mathrm{V}_{4} \mathrm{~S}_{2}$ & 63.47 & 51.63 & 36.41 & 24.54 & 16.39 & 4.80 & 0.00 & 0.00 \\
\hline $\mathrm{V}_{4} \mathrm{~S}_{3}$ & 47.95 & 35.73 & 25.80 & 19.40 & 12.18 & 3.94 & 0.00 & 0.00 \\
\hline $\mathrm{V}_{4} \mathrm{~S}_{4}$ & 9.36 & 0.00 & 0.00 & 0.00 & 0.00 & 0.00 & 0.00 & 0.00 \\
\hline S.Em. \pm & 0.54 & 0.48 & 0.50 & 0.43 & 0.40 & 0.24 & 0.11 & 0.02 \\
\hline C.D. at $5 \%$ & 1.50 & 1.33 & 1.41 & 1.20 & 1.12 & 0.69 & 0.30 & 0.06 \\
\hline C.V. $\%$ & 3.20 & 3.64 & 5.34 & 6.49 & 8.88 & 9.20 & 11.59 & 10.10 \\
\hline
\end{tabular}

Table.5 Combined interaction effect of variety, media and storage temperature on in vitro pollen germination

\begin{tabular}{|c|c|c|c|c|c|c|c|c|c|c|c|c|}
\hline \multicolumn{13}{|c|}{ At 7 days of storage } \\
\hline & \multicolumn{3}{|l|}{$V_{1}$} & \multicolumn{3}{|l|}{$\mathbf{V}_{2}$} & \multicolumn{3}{|l|}{$\mathbf{V}_{3}$} & \multicolumn{3}{|l|}{$V_{4}$} \\
\hline & $\mathbf{M}_{1}$ & $\mathbf{M}_{2}$ & $\mathbf{M}_{3}$ & $\mathbf{M}_{1}$ & $\mathbf{M}_{2}$ & $M_{3}$ & $\mathbf{M}_{1}$ & $\mathbf{M}_{2}$ & $\mathbf{M}_{3}$ & $\mathbf{M}_{1}$ & $\mathbf{M}_{2}$ & $\mathbf{M}_{3}$ \\
\hline $\mathbf{S}_{1}$ & $\underline{85.93}$ & 73.50 & 54.50 & 84.21 & 74.96 & 49.15 & 87.20 & 76.79 & 56.46 & 84.58 & 72.70 & 51.35 \\
\hline $\mathbf{S}_{\mathbf{2}}$ & 81.62 & 73.19 & 43.02 & 76.70 & 64.10 & 39.30 & 78.02 & 71.42 & 51.63 & 75.63 & 67.40 & 47.37 \\
\hline $\mathbf{S}_{3}$ & 80.44 & 74.67 & 35.38 & 66.54 & 61.10 & 24.48 & 69.23 & 63.80 & 32.50 & 63.30 & 57.72 & 22.83 \\
\hline $\mathbf{S}_{4}$ & 25.97 & 19.20 & 0.00 & 13.84 & 5.52 & 0.00 & 29.59 & 17.47 & 0.00 & 16.55 & 11.52 & 0.00 \\
\hline \multicolumn{13}{|c|}{ At 14 days of storage } \\
\hline & \multicolumn{3}{|c|}{$\mathrm{V}_{1}$} & \multicolumn{3}{|l|}{$\mathbf{V}_{2}$} & \multicolumn{3}{|l|}{$\mathbf{V}_{3}$} & \multicolumn{3}{|l|}{$V_{4}$} \\
\hline & $\mathbf{M}_{1}$ & $\mathbf{M}_{2}$ & $\mathbf{M}_{3}$ & $\mathbf{M}_{1}$ & $\mathbf{M}_{2}$ & $\mathbf{M}_{3}$ & $\mathbf{M}_{1}$ & $\mathbf{M}_{2}$ & $\mathbf{M}_{3}$ & $\mathbf{M}_{1}$ & $\mathbf{M}_{2}$ & $\mathbf{M}_{3}$ \\
\hline $\mathbf{S}_{1}$ & 79.61 & 63.71 & 41.25 & 76.13 & 63.28 & 38.07 & 75.27 & 69.79 & 38.86 & 74.67 & 63.38 & 39.61 \\
\hline $\mathbf{S}_{\mathbf{2}}$ & 74.30 & 62.72 & 35.24 & 63.84 & 55.81 & 27.04 & 63.59 & 61.70 & 26.61 & 65.91 & 55.90 & 33.08 \\
\hline $\mathbf{S}_{3}$ & 72.99 & 59.94 & 20.88 & 57.28 & 46.04 & 13.98 & 60.81 & 56.74 & 12.40 & 53.59 & 42.87 & 10.74 \\
\hline $\mathbf{S}_{4}$ & 13.01 & 0.00 & 0.00 & 0.00 & 0.00 & 0.00 & 11.05 & 0.00 & 0.00 & 0.00 & 0.00 & 0.00 \\
\hline \multicolumn{13}{|c|}{ At 21 days of storage } \\
\hline & \multicolumn{3}{|l|}{$V_{1}$} & \multicolumn{3}{|l|}{$\mathbf{V}_{2}$} & \multicolumn{3}{|l|}{$\mathbf{V}_{\mathbf{3}}$} & \multicolumn{3}{|l|}{$V_{4}$} \\
\hline & $\mathbf{M}_{1}$ & $\mathbf{M}_{2}$ & $\mathbf{M}_{3}$ & $\mathbf{M}_{1}$ & $\mathbf{M}_{2}$ & $\mathbf{M}_{3}$ & $\mathbf{M}_{1}$ & $\mathbf{M}_{2}$ & $\mathbf{M}_{3}$ & $\mathbf{M}_{1}$ & $\mathbf{M}_{2}$ & $\mathbf{M}_{3}$ \\
\hline
\end{tabular}




\begin{tabular}{|c|c|c|c|c|c|c|c|c|c|c|c|c|}
\hline $\mathbf{S}_{1}$ & 63.30 & 55.76 & 27.09 & 61.74 & 51.96 & 16.66 & $\underline{63.78}$ & 57.12 & 18.72 & 64.88 & 54.61 & 12.09 \\
\hline $\mathbf{S}_{\mathbf{2}}$ & 59.10 & 51.67 & 15.77 & 52.69 & 43.08 & 0.00 & 56.94 & 51.74 & 14.32 & 51.27 & 46.50 & 11.45 \\
\hline $\mathbf{S}_{\mathbf{3}}$ & 59.82 & 46.98 & 0.00 & 44.29 & 31.40 & 0.00 & 47.24 & 44.71 & 0.00 & 43.12 & 34.27 & 0.00 \\
\hline $\mathbf{S}_{4}$ & 0.00 & 0.00 & 0.00 & 0.00 & 0.00 & 0.00 & 0.00 & 0.00 & 0.00 & 0.00 & 0.00 & 0.00 \\
\hline \multicolumn{13}{|c|}{ At 28 days of storage } \\
\hline & \multicolumn{3}{|l|}{$\mathbf{V}_{1}$} & \multicolumn{3}{|l|}{$V_{2}$} & \multicolumn{3}{|l|}{$\mathbf{V}_{\mathbf{3}}$} & \multicolumn{3}{|l|}{$\mathrm{V}_{4}$} \\
\hline & $\mathbf{M}_{1}$ & $\mathbf{M}_{2}$ & $\mathbf{M}_{3}$ & $\mathbf{M}_{1}$ & $\mathbf{M}_{2}$ & $\mathbf{M}_{3}$ & $\mathbf{M}_{1}$ & $\mathbf{M}_{2}$ & $\mathbf{M}_{3}$ & $\mathbf{M}_{1}$ & $\mathbf{M}_{2}$ & $\mathbf{M}_{3}$ \\
\hline $\mathbf{S}_{1}$ & 50.08 & 44.40 & 12.30 & 49.46 & 39.25 & 0.00 & 54.79 & 46.45 & 9.66 & $\underline{53.44}$ & 39.04 & 0.00 \\
\hline $\mathbf{S}_{2}$ & 52.90 & 38.50 & 0.00 & 33.40 & 23.83 & 0.00 & 41.45 & 33.97 & 0.00 & 42.09 & 31.54 & 0.00 \\
\hline $\mathbf{S}_{3}$ & 45.90 & 32.69 & 0.00 & 30.08 & 20.90 & 0.00 & 33.86 & 33.11 & 0.00 & 34.81 & 23.38 & 0.00 \\
\hline $\mathbf{S}_{4}$ & 0.00 & 0.00 & 0.00 & 0.00 & 0.00 & 0.00 & 0.00 & 0.00 & 0.00 & 0.00 & 0.00 & 0.00 \\
\hline \multicolumn{13}{|c|}{35 days of storage } \\
\hline & \multicolumn{3}{|l|}{$\mathbf{V}_{1}$} & \multicolumn{3}{|l|}{$V_{2}$} & \multicolumn{3}{|l|}{$V_{3}$} & \multicolumn{3}{|l|}{$V_{4}$} \\
\hline & $\mathbf{M}_{1}$ & $\mathbf{M}_{2}$ & $\mathbf{M}_{3}$ & $\mathbf{M}_{1}$ & $\mathbf{M}_{2}$ & $\mathbf{M}_{3}$ & $\mathbf{M}_{1}$ & $\mathbf{M}_{2}$ & $\mathbf{M}_{3}$ & $M_{1}$ & $\mathbf{M}_{2}$ & $\mathbf{M}_{3}$ \\
\hline $\mathbf{S}_{1}$ & $\underline{46.66}$ & 31.26 & 0.00 & 32.82 & 23.41 & 0.00 & 47.03 & 32.45 & 0.00 & 41.68 & 24.48 & 0.00 \\
\hline $\mathbf{S}_{2}$ & 30.53 & 23.07 & 0.00 & 21.42 & 14.63 & 0.00 & 33.15 & 23.13 & 0.00 & 29.48 & 19.70 & 0.00 \\
\hline $\mathbf{S}_{3}$ & 32.18 & 28.79 & 0.00 & 23.18 & 9.13 & 0.00 & 25.33 & 19.18 & 0.00 & 23.84 & 12.71 & 0.00 \\
\hline $\mathbf{S}_{4}$ & 0.00 & 0.00 & 0.00 & 0.00 & 0.00 & 0.00 & 0.00 & 0.00 & 0.00 & 0.00 & 0.00 & 0.00 \\
\hline \multicolumn{13}{|c|}{42 days of storage } \\
\hline & \multicolumn{3}{|l|}{$\mathbf{V}_{1}$} & \multicolumn{3}{|l|}{$V_{2}$} & \multicolumn{3}{|l|}{$\mathbf{V}_{3}$} & \multicolumn{3}{|l|}{$V_{4}$} \\
\hline & $\mathbf{M}_{1}$ & $\mathbf{M}_{2}$ & $\mathbf{M}_{3}$ & $\mathbf{M}_{1}$ & $\mathbf{M}_{2}$ & $\mathbf{M}_{3}$ & $\mathbf{M}_{1}$ & $\mathbf{M}_{2}$ & $\mathbf{M}_{3}$ & $\mathbf{M}_{1}$ & $\mathbf{M}_{2}$ & $\mathbf{M}_{3}$ \\
\hline $\mathbf{S}_{1}$ & 36.33 & 27.92 & 0.00 & 22.27 & 13.90 & 0.00 & $\underline{35.83}$ & 23.01 & 0.00 & 33.08 & 11.58 & 0.00 \\
\hline $\mathbf{S}_{2}$ & 26.41 & 15.63 & 0.00 & 14.07 & 8.81 & 0.00 & 19.23 & 14.58 & 0.00 & 14.41 & 0.00 & 0.00 \\
\hline $\mathbf{S}_{\mathbf{3}}$ & 19.13 & 10.65 & 0.00 & 11.46 & 0.00 & 0.00 & 11.75 & 0.00 & 0.00 & 11.81 & 0.00 & 0.00 \\
\hline $\mathbf{S}_{4}$ & 0.00 & 0.00 & 0.00 & 0.00 & 0.00 & 0.00 & 0.00 & 0.00 & 0.00 & 0.00 & 0.00 & 0.00 \\
\hline \multicolumn{13}{|c|}{49 days of storage } \\
\hline & \multicolumn{3}{|l|}{$\mathbf{V}_{1}$} & \multicolumn{3}{|l|}{$\mathbf{V}_{2}$} & \multicolumn{3}{|l|}{$\mathbf{V}_{3}$} & \multicolumn{3}{|l|}{$\mathrm{V}_{4}$} \\
\hline & $\mathbf{M}_{1}$ & $\mathbf{M}_{2}$ & $\mathbf{M}_{3}$ & $\mathbf{M}_{1}$ & $\mathbf{M}_{2}$ & $\mathbf{M}_{3}$ & $\mathbf{M}_{1}$ & $\mathbf{M}_{2}$ & $\mathbf{M}_{3}$ & $\mathbf{M}_{1}$ & $\mathbf{M}_{2}$ & $\mathbf{M}_{3}$ \\
\hline $\mathbf{S}_{1}$ & 24.69 & 12.20 & 0.00 & 10.69 & 0.00 & 0.00 & 24.16 & 14.83 & 0.00 & 25.49 & 0.00 & 0.00 \\
\hline $\mathbf{S}_{2}$ & 10.80 & 0.00 & 0.00 & 0.00 & 0.00 & 0.00 & 9.88 & 0.00 & 0.00 & 0.00 & 0.00 & 0.00 \\
\hline $\mathbf{S}_{3}$ & 0.00 & 0.00 & 0.00 & 0.00 & 0.00 & 0.00 & 0.00 & 0.00 & 0.00 & 0.00 & 0.00 & 0.00 \\
\hline $\mathbf{S}_{4}$ & 0.00 & 0.00 & 0.00 & 0.00 & 0.00 & 0.00 & 0.00 & 0.00 & 0.00 & 0.00 & 0.00 & 0.00 \\
\hline \multicolumn{13}{|c|}{56 days of storage } \\
\hline & \multicolumn{3}{|l|}{$V_{1}$} & \multicolumn{3}{|l|}{$\mathbf{V}_{2}$} & $\mathbf{V}_{3}$ & & & $V_{4}$ & & \\
\hline & $\mathbf{M}_{1}$ & $\mathbf{M}_{2}$ & $\mathbf{M}_{3}$ & $\mathbf{M}_{1}$ & $\mathbf{M}_{2}$ & $\mathbf{M}_{3}$ & $\mathbf{M}_{1}$ & $\mathbf{M}_{2}$ & $\mathbf{M}_{3}$ & $\mathbf{M}_{1}$ & $\mathbf{M}_{2}$ & $\mathbf{M}_{3}$ \\
\hline $\mathbf{S}_{1}$ & 11.45 & 0.00 & 0.00 & 0.00 & 0.00 & 0.00 & 9.42 & 0.00 & 0.00 & 10.35 & 0.00 & 0.00 \\
\hline $\mathbf{S}_{\mathbf{2}}$ & 0.00 & 0.00 & 0.00 & 0.00 & 0.00 & 0.00 & 0.00 & 0.00 & 0.00 & 0.00 & 0.00 & 0.00 \\
\hline $\mathbf{S}_{3}$ & 0.00 & 0.00 & 0.00 & 0.00 & 0.00 & 0.00 & 0.00 & 0.00 & 0.00 & 0.00 & 0.00 & 0.00 \\
\hline $\mathbf{S}_{4}$ & 0.00 & 0.00 & 0.00 & 0.00 & 0.00 & 0.00 & 0.00 & 0.00 & 0.00 & 0.00 & 0.00 & 0.00 \\
\hline
\end{tabular}

\begin{tabular}{|l|c|c|c|c|c|c|c|c|}
\hline Days of storage & $\mathbf{7}$ & $\mathbf{1 4}$ & $\mathbf{2 1}$ & $\mathbf{2 8}$ & $\mathbf{3 5}$ & $\mathbf{4 2}$ & $\mathbf{4 9}$ & $\mathbf{5 6}$ \\
\hline S.Em. \pm & 0.93 & 0.82 & 0.87 & 0.74 & 0.69 & 0.42 & 0.19 & 0.04 \\
\hline C.D. at 5\% & 2.61 & 2.31 & 2.44 & 2.08 & 1.95 & 1.19 & 0.52 & 0.11 \\
\hline C.V. \% & 3.20 & 3.64 & 5.34 & 6.49 & 8.88 & 9.20 & 11.59 & 10.10 \\
\hline
\end{tabular}


Minimum pollen germination was observed in $\mathrm{M}_{2} \mathrm{~S}_{4}$ at 7 days which turned to zero from 14 days of storage.

\section{Interaction effect of variety and storage temperature on in vitro pollen germination}

Similarly, variation due to the interaction effect of variety and storage temperature was also found significant and maximum pollen germination $(73.49,36.97,26.49 \& 13.23 \%)$ was noted in $\mathrm{V}_{3} \mathrm{~S}_{1}$ during $7,28,35$ and 49 days of storage. Elsewhere at 14, 21, 42 and 56 days of storage, maximum pollen germination $(61.52,48.72,21.42 \& 3.82 \%)$ were noted in $\mathrm{V}_{1} \mathrm{~S}_{1}$. Similar trend was observed here also and Kesar and Mallika showed good pollen germination at $-20^{\circ} \mathrm{C}$ might be due to low metabolic activities of pollen at sub-zero storage temperature. Minimum pollen germination was noted in variety Alphonso stored at room temperature $\left(\mathrm{V}_{2} \mathrm{~S}_{4}\right)$ at 7 days of storage but from 14 days of storage, it turns to zero germination in all varieties (Table 4).

Combined interaction effect of variety, media and storage temperature on in vitro pollen germination

Due to combined interaction effect of variety, media and storage temperature, the wide variation for pollen germination was also observed significant at all days of storage. Maximum pollen germination $(87.20,54.79$ and $47.03 \%$ ) was noted in $\mathrm{V}_{3} \mathrm{M}_{1} \mathrm{~S}_{1}$ at 7,28 and 35 days of storage. Similarly at 14 and 42 days, it (79.61 and $36.33 \%$ ) was noted in $\mathrm{V}_{1} \mathrm{M}_{1} \mathrm{~S}_{1}$ while at 21 and 49 days, it (64.88 and $25.49 \%$ ) was noted in $\mathrm{V}_{4} \mathrm{M}_{1} \mathrm{~S}_{1}$. At 56 days of storage, all treatment combinations were recorded with zero per cent pollen germination except $\mathrm{V}_{1} \mathrm{M}_{1} \mathrm{~S}_{1}(11.45 \%)$ which was highest. Likewise, lowest pollen germination was noted in $\mathrm{V}_{2} \mathrm{M}_{2} \mathrm{~S}_{4}, \mathrm{~V}_{4} \mathrm{M}_{3} \mathrm{~S}_{3}$, $\mathrm{V}_{4} \mathrm{M}_{3} \mathrm{~S}_{2}$ and $\mathrm{V}_{1} \mathrm{M}_{3} \mathrm{~S}_{1}$ at 7, 14, 21 and 28 days of storage, respectively. Similarly, pollen grains stored without media at room temperature showed no germination for all varieties from 7 days (Table 5).

\section{Effect of variety on in vitro pollen germination}

The pollen germination differed depending on storage temperature, storage duration and cultivar. The differences in germination rates for the genotypes and cultivars might be due to genotype differences. The result was in confirming with those by Khan and Perveen (2009), Abourayya et al., (2011) and Dutta et al., (2013) in mango. Present finding were also in agreement with the results reported by Sahar and Spiegel (1984), Alburquerque et al., (2007), Sharafi and Bahmani (2011), Bhat et al., (2012), Gaaliche et al., (2013), Marlon et al., (2014), Khan and Perveen (2014) and Ahmed et al., (2017) in avocado, sweet cherry, almond, pear, fig, custard apple and citrus, respectively.

\section{Effect of media on in vitro pollen germination}

The variation observed in pollen germination due to storage media was also found significant and might be due to the sensitivity of mango pollen to extended storage media exposure. However, decreased pollen germination from exposure to organic solvents may be the result of leaching of phospholipids, sugars, and amino acids from the stored pollen.

The loss of these compounds compromises pollen membrane integrity and consequently affects pollen germination (Jain and Shivanna, 1988). Results were also in close agreements with the finding of Kumar et al., (2015) as well as with Jain and Shivanna (1988, 1990) in oil palm and crotalaria, respectively. 
Effect of storage temperature on in vitro pollen germination

Wide differences in germination of pollen grains were observed significant with different storage temperatures and durations among different mango cultivars. Higher values for pollen germination at low storage temperature and decline at high storage temperature were observed by several workers (Khan and Perveen, 2008). The rate of reduction in pollen germination might be because of differential rate of metabolic activities in pollen at different temperature. From 21 days of storage, pollen grain stored at room temperature was completely lost its germination capacity. Our results clearly indicated that it is feasible to store pollen grains of mango at sub-zero temperatures ($20^{\circ} \mathrm{C}$ ) without any significant loss in their germination capacity which was confirming with Bhat et al., (2012) in pear. Present results were also in agreement with Salles et al., (2007), Thaipong et al., (2008) and Chander et al., (2019) in citrus, grape and sugar apple, respectively.

Interaction effect of variety and media on in vitro pollen germination

Variation due to interaction effect of variety and media on pollen germination was found significant and might be due to the different genotype of the varieties and their interaction with storage media. Kesar had good pollen germination ability while stored in n-hexane could be due to very little leaching of sugars and amino acids into the solvent for good germination.

Interaction effect of media and storage temperature on in vitro pollen germination

Variation due to interaction effect of different media and storage temperatures on pollen germination was also found significant. As mango pollen was more sensitive to desiccation and high temperature, zero pollen germination was noticed in pollen grains stored without media at room temperature $\left(\mathrm{M}_{3} \mathrm{~S}_{4}\right)$ from 7 days of storage. Results were also in agreement with Khan and Perveen (2008).

Interaction effect of variety and storage temperature on in vitro pollen germination

Similarly, variation due to the interaction effect of variety and storage temperature was also found significant. Kesar and Mallika showed good pollen germination at $-20^{\circ} \mathrm{C}$ might be due to low metabolic activities of pollen at sub-zero storage temperature. Minimum pollen germination was noted in variety Alphonso stored at room temperature $\left(\mathrm{V}_{2} \mathrm{~S}_{4}\right)$ at 7 days of storage but from 14 days of storage, it turns to zero germination in all varieties. The decrease in pollen germination capacity at room temperature might be due to high sensitivity of mango pollen to existing high temperatures. However, results from this work were in agreement with those found by Alburquerque et al., (2007), who found a highly significant effect of pollen genotype and temperature. Higher temperature reduced pollen germination. The result was also confirming with Imani et al., (2011) and Bhat et al., (2012) in apple and pear, respectively.

Combined interaction effect of variety, media and storage temperature on in vitro pollen germination

Due to combined interaction effect of variety, media and storage temperature, the wide variation for pollen germination was also observed significant at all days of storage. Results were observed due to different genotype of varieties, different nature of storage media and different metabolic activity rates of pollen at different storage temperature. Due to tolerability of Kesar 
pollen against various storage conditions, less leaching of metabolites in n-hexane and very low metabolic activities at sub-zero $\left(-20^{\circ} \mathrm{C}\right)$ storage temperature, its pollen could be stored more successfully up to 56 days compared to others.

On the basis of results obtained from the present investigation, it can be concluded that germination ability of mango pollen grains could be retained successfully in different storage media at low temperature up to 56 days. Among four varieties tested, Kesar and Mallika recorded as good in terms of pollen germination capacity. In storage media, nhexane was found best in terms of retaining more germination ability in all four varieties but on the other hand success rate was found minimum in case of no media. Storage at $20^{\circ} \mathrm{C}$ gave better results in terms of in vitro pollen germination under study. Therefore, the best suitable temperature for long duration of pollen storage would be $-20^{\circ} \mathrm{C}$. At room temperature without media, pollen grains could not be stored more than a week due to rapid decrease in their germination capacity. Due to that, pollen grains stored without media at room temperature cannot be adopted. In case of interactions, pollen grains of Kesar, Mallika and Dudhpendo stored in n-hexane at $-20^{\circ} \mathrm{C}$ were found best in terms of pollen germination up to 56 days.

\section{Acknowledgement}

I am indebted to my major and minor advisors, Dr. D. K. Varu and Dr. D. R. Mehta for their valuable support during whole research program. With full honors and ecstasy of delight, I express my heart felt and special thanks for the facilities provided by Dr. V. P. Chovatia, Hon'ble Vice Chancellor, and Director of Research and Dean P. G. Studies, Junagadh Agricultural University, Junagadh.

\section{References}

Abourayya, M.S., Kassim N.E., El-Sheikh N.E. and Rakha, A.M. 2011. Comparative study between inflorescence characteristics, pollen viability, germination and dimensions of Tommy Atkins, Kent and Keitt mango cultivars. Life Sci. J., 8(1): 100105.

Ahmed, S., Rattanpal H.S., Ahmad E. and Singh, G. 2017. Influence of storage duration and storage temperature on in vitro pollen germination of citrus species. Int. J. Curr. Microbiol. App. Sci., 6(5): 892-902.

Alburquerque, N., Montiel, F.G. and Burgos, L. 2007. Influence of storage temperature on the viability of sweet cherry pollen. Span J Agric Res., 5(1): 86-90.

Bhat, Z.A., Dhillon, W.S., Shafi, R.H.S., Rather, J.A., Mir, A.H., Shafi, W., Rashid, R., Bhat, J.A., Rather, T.R. and Wani, T.A. 2012. Influence of storage temperature on viability and in vitro germination capacity of pear (Pyrus spp.) Pollen. J. of Agril. Sci., 4(11): 128-135.

Chander, S., Rajasekharan, P.E. and Kurian, R.M. 2019. Pollen storage studies in sugar apple (Annona squamosa L.) cv. Balanagar, Israel J. of Pl. Sci., 66(4): 196-202.

Davenport, T.L. 2009. Reproductive physiology. In: Litz, R.E. (Ed.), The Mango: Botany, Production and Uses., 2nd ed. CAB International, Wallingford, UK, pp. 97-169.

De Wet, E. and Robbertse, P.J. 1986. The influence of temperature and boron on pollen germination in Mangifera indica L. S. Afr. J. Plant Soil., 6: 228-234.

Dutta, S.K., Srivastav, M., Rymbai, H., Chaudhary, R., Singh, A.K., Dubey, A.K. and Lal, K. 2013. Pollen-pistil 
interaction studies in mango (Mangifera indica L.) cultivars. Sci. Hortic., 160: 213-221.

Free, J. B. 1993. Insect Pollination of Crops, second edition. Academic Press, London.

Gaaliche, B., Majdoub, A., Trad, M. and Mars, M. 2013 Assessment of pollen viability, germination, and tube growth in eight Tunisian Caprifig (Ficus carica L.) cultivars. Hindawi Publishing Corporation.

Galan-Sauco, V. 1999. El cultivo del mango. Ediciones Mundi-Prensa, Barcelona.

Imani, A., Barzegar, K., Piripireivatlou, S. and Masomi, S.H. 2011. Storage of apple pollen and in vitro germination. African J. of Agril. Res., 6(2): 624-629.

Iyler, C.P.A. and Scnell, R.J. 2009. Breeding and genetics. In: Litz, R. E. (Ed.), The Mango: Botany, Production and Uses. $2^{\text {nd }}$ edition. $C A B$ International, Wallingford, UK, pp. 67-96.

Jain, A. and Shivanna, K.R. 1988. Storage of pollen grains in organic solvents: effects of solvents on pollen viability and membrane integrity. J. of Pl. Physi., 132: 499-501.

Jain, A. and Shivanna, K.R. 1990. Storage of pollen grains of Crotalaria retusa in oils. Sexual Pl. Repro., 3: 225-227.

Khan, S.A. and Perveen, A. 2008. Maintenance of pollen germination capacity of Malus pumila L., (Rosaceae). Pak. J. Bot., 40(3): 963966.

Khan, S.A. and Perveen, A. 2009. Pollen germination capacity of three mango cultivars (Mangifera indica L., Anacardiaceae) from Pakistan. Pak. J. Bot., 41(3): 1009-1012.

Khan, S.A. and Perveen, A. 2014. In vitro pollen germination of five citrus species. Pak. J. Bot., 46(3): 951-956

Kosterman, A.J. and Bompard, J.M. 1993. The mangoes: their botany, nomenclature, horticulture and utilization. Academic Press, London, UK.

Kumar, K.S., Mathur, R.K. and Sparjanbabu, D.S. 2015. Efficacy of organic solvents for medium term storage of oil palm (Elaeis guineensis Jacq.) pollen. Ind. J. Agric. Res., 49 (6): 516-521.

Marlon, C.T., Pereira, Jonathan, H., Crane, Montas, W., Nietsche, S., Wagner and Vendrame, A. 2014. Effects of storage length and flowering stage of pollen influence its viability, fruit set and fruit quality in 'Red' and 'Lessard Thai' sugar apple (Annona squamosa) and 'Gefner' atemoya (A. cherimola $\times A$. squamosa). Sci. Hortic., 178: 55-60

Naik, K.C. and Rao, M.M. 1943. Studies on the blossom biology and pollination in mangoes (Mangifera indica L.). Indian J. Hortic., 1: 107-119.

Panse, V.G. and Sukhatme, P.V. 1985. Statistical Method for Agricultural Workers. ICAR publ., New Delhi, p. 361.

Pimentel, R.B., Coronel, R.E., Espino, R.F.C. 1984. Floral biology and fruit set in mango (Mangifera indica L.) cultivars: 'Carabao', 'Pico' and 'Kancha Mitha'. Phillippine J. Crop Sci., 9: 47-51.

Sahar, N. and Spiegel, R. 1984. In vitro germination of avocado pollen. Hort. Science, 19(6): 886-888.

Salles, P.L.A., Darlan, R.J., Junqueira, P.M., Santos, K.P. and Morais, J.C. 2007. Viability of citrus pollen in different storage conditions. Ciência $e$ Agrotecnologia, 31(1), 147-153.

Scholefield, P.B. 1982. Scanning electron microscope study of flowers of avocado, litchi, macadamia and mango. Sci. Hortic., 16: 263-272.

Sen, P.K., Mallik, P.C. and Ganguly, B.D. 1946. Hybridization of the mango. Indian J. Hortic. 4: 4-15.

Sharafi, Y. and Bahmani, B. 2011. In vitro 
study of pollen traits after short storage in some almond, apricot and sweet cherry favorable genotypes. J. of Med. Pl. Res., 5(2): 266-269.

Singh, R.N. 1954. Studies in floral biology and subsequent development of fruits in the mango (Mangifera indica L.) Dasheri and Langra. Indian J. Horti., 11(3): 69-88.

Singh, S.N. 1961. Studies on the morphology and viability of the pollen grains of mango. Hortic. Adv., 5: 121-144.

Spencer, J.L. and Kennard, W.C. 1955. Studies on mango fruit set in Puerto Rico. Trop. Agri., 32: 323-330.

Thaipong, K., Dalaeman, S. and Sethpakdee, R. 2008. An appropriate temperature for grape pollen storage. Agril. Sci. J., 39(3): 36-39.

\section{How to cite this article:}

Dhamsaniya, D. N., D. K. Varu and Mehta, D. R. 2020. Effect of Different Varieties, Media and Storage Temperature on In vitro Pollen Germination in Mango (Mangifera indica L.). Int.J.Curr.Microbiol.App.Sci. 9(10): 569-580. doi: https://doi.org/10.20546/ijcmas.2020.910.068 